\title{
Hashimoto's Encephalopathy Presenting with Smoldering Limbic Encephalitis
}

\author{
Masahiro Nagano ${ }^{1}$, Katsuya Kobayashi ${ }^{1}$, Mayumi Yamada-Otani ${ }^{1}$, Akira Kuzuya ${ }^{1}$, \\ Riki Matsumoto ${ }^{1}$, Jiro Oita ${ }^{2}$, Makoto Yoneda ${ }^{3}$, Akio Ikeda ${ }^{4}$ and Ryosuke Takahashi ${ }^{1}$
}

\begin{abstract}
:
Hashimoto's encephalopathy (HE) is a steroid-responsive autoimmune encephalopathy associated with Hashimoto thyroiditis. We herein report a case of HE manifesting "smoldering" limbic encephalitis with persisting symptoms and abnormalities on examinations. Although our patient experienced partial clinical remission after treatment, hippocampal hypermetabolism on $\left[{ }^{18} \mathrm{~F}\right]$ fluorodeoxyglucose positron emission tomography (FDG-PET) and subclinical seizures on video electroencephalography persisted. Hypermetabolism on FDG-PET was improved by additional prednisolone therapy. Thus, as with other autoimmune limbic encephalitis cases, HE can take a course of "smoldering" encephalitis. FDG-PET and electroencephalogram findings can reflect the disease activity degree in such patients, although with certain neurophysiological and biochemical distinctions.
\end{abstract}

Key words: Hashimoto's encephalopathy, FDG-PET, video EEG, autoimmune limbic encephalitis, anti-NAE antibody

(Intern Med 58: 1167-1172, 2019)

(DOI: 10.2169/internalmedicine.1289-18)

\section{Introduction}

Hashimoto's encephalopathy (HE) is an encephalopathy related to thyroid autoimmunity and was first described by Brain et al. (1). HE has various clinical characteristics, but the precise pathomechanism has not been elucidated (2). Although $\mathrm{HE}$ is a relatively rare disease, it is sometimes misdiagnosed as other neurological disorders, such as Alzheimer's disease, schizophrenia, and depression, or goes undiagnosed, due to its various manifestations (3-6). Yoneda et al. revealed that serum anti-NH2 terminal of $\alpha$-enolase (NAE) antibody was a specific biomarker of HE and that a subtype of HE sometimes showed limbic encephalitis, namely antiNAE antibody limbic encephalitis $(7,8)$. We previously reported a case of anti-leucine-rich glioma inactivated 1 protein (LGI1) antibody encephalitis in which hypermetabolism on $\left[{ }^{18} \mathrm{~F}\right]$ fluorodeoxyglucose positron emission tomography (FDG-PET) and subclinical seizure patterns on video elec- troencephalogram (vEEG) chronically persisted, leading us to term the disorder "smoldering" encephalitis $(9,10)$. In autoimmune encephalitis, hypermetabolism on FDG-PET has generally been considered to reflect the degree of inflammation, which can be a surrogate marker for its treatment (11-15).

We herein report a case of HE presenting with "smoldering" limbic encephalitis. Both FDG-PET and long-term vEEG were useful for evaluating the disease activity.

\section{Case Report}

A 68-year-old Japanese woman without a remarkable medical history developed a 1-week history of memory impairment, resulting in some trouble at her workplace. She appeared mostly normal, but her activities of daily living, such as cooking, were slightly impaired. She became confused after a few days and then developed somnolence, at which point she was admitted to a hospital.

\footnotetext{
${ }^{1}$ Department of Neurology, Kyoto University Graduate School of Medicine, Japan, ${ }^{2}$ Department of Neurology, Shimizu Hospital, Japan, ${ }^{3}$ Faculty of Nursing and Social Welfare Sciences, Fukui Prefectural University, Japan and ${ }^{4}$ Department of Epilepsy, Movement Disorders and Physiology, Kyoto University Graduate School of Medicine, Japan

Received for publication March 30, 2018; Accepted for publication October 8, 2018

Correspondence to Dr. Katsuya Kobayashi, 31258a@kuhp.kyoto-u.ac.jp
} 
Onset

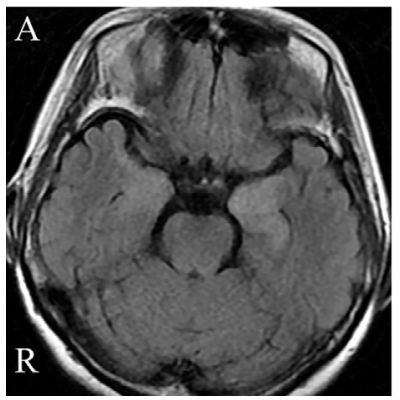

4 months after onset

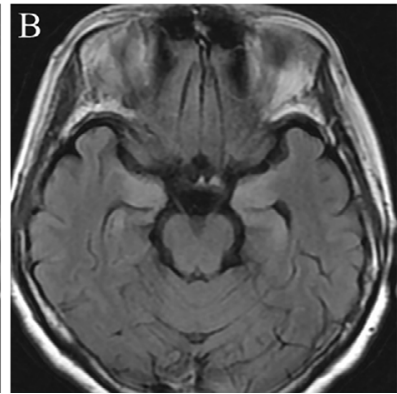

7 months after onset

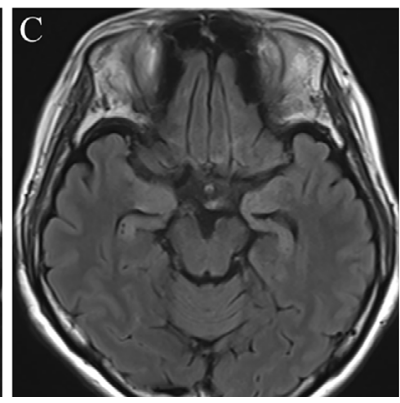

12 months after onset

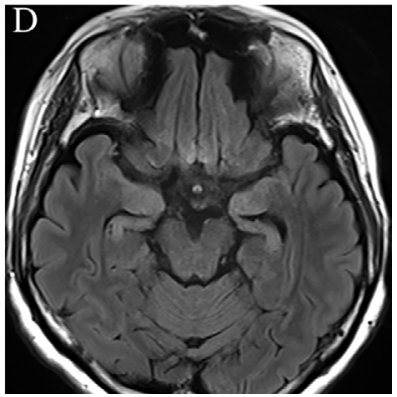

Figure 1. Chronological changes in brain MRI. (A) At the initial attack, brain magnetic resonance imaging (MRI) on fluid-attenuated inversion recovery (FLAIR) showed enlargement of bilateral medial temporal lobes with high-intensity signals. (B) At the second attack, MRI showed hyperintense lesions in the bilateral medial temporal lobe with slight atrophy. (C) Seven months after the onset, MRI showed bilateral hippocampal atrophy with high-intensity signals. (D) Twelve months after the onset, MRI showed no remarkable interval changes from $(C)$.

A neurological examination revealed severe consciousness disturbance that precluded the Mini-Mental State Examination (MMSE) evaluation. The findings of a general blood examination were within normal limits. Thyroid-stimulating hormone (TSH), free thyroxine (T4) and free triiodothyronine (T3) were almost within normal limits $(0.57 \mu \mathrm{IU} / \mathrm{mL}$; normal $0.34-3.88 \mu \mathrm{IU} / \mathrm{mL}$, and $1.2 \mathrm{ng} / \mathrm{dL}$; normal $0.8-1.8$ $\mathrm{ng} / \mathrm{dL}, 2.0 \mathrm{pg} / \mathrm{dL}$; normal 2.1-4.0 pg/mL, respectively). A cerebrospinal fluid (CSF) analysis showed mildly elevated protein (44 mg/dL) and lymphocyte dominant pleocytosis (24 cells $/ \mathrm{mm}^{3}, 96 \%$ monocytes). Brain magnetic resonance imaging (MRI) showed bilateral high-intensity lesions in the hippocampus and amygdala on fluid-attenuated inversion recovery (FLAIR) imaging (Fig. 1A). Whole-body contrastenhanced computed tomography (CT) showed no mass lesion indicating a suspected malignant tumor. An electroencephalogram (EEG) showed spike and wave complexes and periodic discharge patterns in the bilateral temporal areas.

We considered some type of viral encephalitis including human herpes virus-6 (HHV-6) in the differential diagnosis, given the patient's immunocompetent background, however, the most likely diagnosis was considered to be herpes simplex virus encephalitis. We therefore started acyclovir and fosphenytoin, which did not improve her symptoms. We ruled out the possibility of a subacute clinical manifestation of a concurrent condition, such as hypothyroidism of degenerative diseases, including Alzheimer's disease, as the cause of her memory disturbance based on the evidence of a normal thyroid function and her clinical background. In addition, nonconvulsive status epilepticus (NCSE) was considered as a differential diagnosis, but there was no fluctuation in her symptoms nor any improvement in her condition with anti-epileptic medication.

Several days after admission, the initial serology was positive for antithyroid peroxidase (anti-TPO) antibody (495 $\mathrm{IU} / \mathrm{mL}$; normal $<16 \mathrm{IU} / \mathrm{mL}$ ) and negative for DNA of herpes simplex virus-1. Considering the possibility of $\mathrm{HE}$, we started intravenous methylprednisolone (IVMP) at 1,000 mg/ day for 3 consecutive days followed by oral glucocorticosteroid (GCS) at $30 \mathrm{mg} /$ day for 8 days. She showed marked cognitive improvement with an Mini-Mental State Examination (MMSE) score of 24/30 compared with a score of 18/30 just before treatment, but mild memory impairment remained. Repeated MRI revealed partial attenuation of the high-intensity signals in the bilateral mesial temporal lobes, and an EEG likewise showed moderate improvement. We finally diagnosed her with HE, as IVMP and oral GCS dramatically improved her symptoms. We continued levetiracetam because the EEG showed epileptiform discharges in the bilateral temporal areas. The patient was discharged 2 months after the onset of initial symptoms with an MMSE score of $28 / 30$.

Two months later, she gradually developed fatigue, daytime sleepiness, and exacerbation of memory impairment. A physical examination showed both consciousness and cognitive impairment with an MMSE score of 25/30. Brain MRI showed bilateral hippocampal high-intensity lesions (Fig. 1B), but an EEG showed no epileptiform discharges. She was diagnosed with a relapse of HE, and IVMP at $1,000 \mathrm{mg} /$ day for 3 days was administered, followed by oral GCS at $30 \mathrm{mg} /$ day for 9 days. Her consciousness disturbance was improved, and she was discharged with memory impairment with an MMSE score of 26/30.

Three months later, she was admitted to the hospital for a further evaluation since her memory impairment had remained without improvement. A neurological examination revealed mild memory impairment on the MMSE (score of 27/30) and the Wechsler Memory Scale-Revised (WMS-R) (verbal memory: 66, visual memory: 88 , general memory: 70, attention: 101, and delayed memory: <50). A laboratory examination was normal except for positive findings for anti-TPO antibody $(37.9 \mathrm{IU} / \mathrm{mL})$, while all other serological tests, including anti-thyrogloblin antibody $(<10.0 \mathrm{IU} / \mathrm{mL}$; normal $<28.0 \mathrm{IU} / \mathrm{mL}$ ), were negative. A CSF examination revealed a mild elevation in protein $(56.6 \mathrm{mg} / \mathrm{dL},>11$ cells/ $\mathrm{mm}^{3}$, IgG index 0.47), while bacterial culture and cytology 
Before steroid therapy

(7 months after onset)

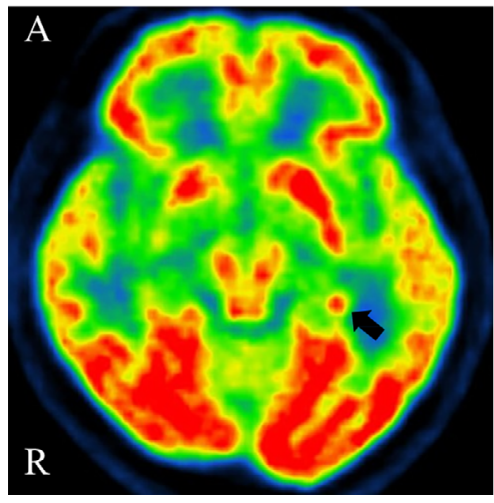

2 weeks after steroid therapy

(7.5 months after onset)

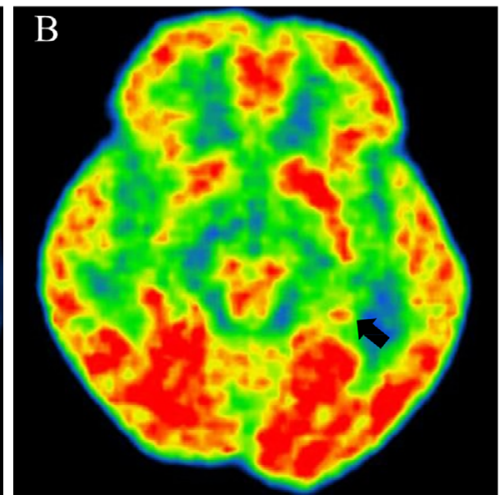

5 months after steroid therapy

(12 months after onset)

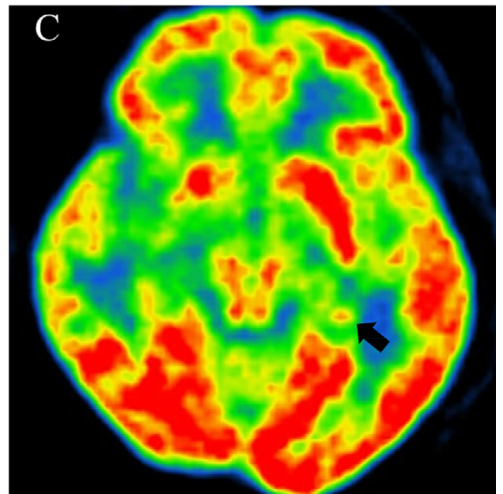

Figure 2. Sequential changes on fluorodeoxyglucose-positron emission tomography (FDG-PET). Fluorodeoxyglucose-positron emission tomography (FDG-PET) showing hypermetabolism in a left posterior hippocampal lesion [standardized uptake value (SUV): mean 6.26, max 7.29] (A), which improved after steroid treatment (SUV: mean 4.38, max 4.96) (B). Image showing that, five months after the initiation of steroid therapy, the improvement in the hypermetabolism had been maintained (SUV: mean 4.38, max 5.04) (C). The SUV values were measured in the region of interest (ROI) including the highest SUV value.

of the CSF were negative. Brain MRI on FLAIR imaging showed bilateral hippocampal high intensity lesions and atrophy (Fig. 1C). FDG-PET showed hypermetabolism in the left posterior hippocampus (Fig. 2A). In order to evaluate the degree of hypermetabolism on FDG-PET suggesting inflammation objectively and chronologically, we measured the standardized uptake value (SUV) of the region of interest (ROI) including the highest SUV value in FDG-PET. At the time of the initial FDG-PET scan, the mean SUV was 6.26, and the maximum SUV was 7.29. An overnight vEEG revealed six subclinical seizures arising from the right midposterior temporal area and one from the left front-mid temporal area. Given the positive anti-TPO antibody result and steroid responsiveness, as well as the hypermetabolism on FDG-PET and subclinical seizures of the bilateral temporal areas on vEEG, we diagnosed the patient HE manifesting as "smoldering" limbic encephalitis.

We introduced IVMP at 1,000 mg/day for 3 consecutive days followed by oral GCS at $25 \mathrm{mg}$ /day. FDG-PET after additional IVMP treatment showed remarkable improvement of hypermetabolism of the left hippocampus (Fig. 2B). We measured the SUV on FDG-PET, including the maximum value with the same-size ROI as at the initial measurement. The mean SUV was 4.38, and the maximum SUV was 4.96, suggesting an improvement in hypermetabolism compared with that before additional IVMP therapy, although the patient's memory impairment was unchanged.

One month later, her serum tested positive for anti-NAE antibody, with negative findings for anti-voltage-gated potassium channel-complex (VGKC-complex) autoantibody, including anti-LGI1 antibody and anti-contactin-associated protein 2 (CAPSR-2) antibody; anti-glutamate receptor $\varepsilon 2$, $\zeta 1$, and $\delta 2$ antibody; anti-Ma1 antibody; anti-Ma2 antibody; anti-amphiphysin antibody; anti-CV2/collapsing response mediator protein 5 (CRMP5) antibody; anti-Ri antibody; anti-Yo antibody; and anti-HuD antibody. We did not measure anti-gamma-aminobutyric acid (GABA)-A/B receptor antibodies. TSH, fT4, and fT3 were all within normal limits (1.52 $\mu \mathrm{IU} / \mathrm{mL}, 1.21 \mathrm{ng} / \mathrm{dL}$, and $3.2 \mathrm{pg} / \mathrm{dL}$, respectively). The dosage of oral GCS was gradually reduced.

Six months later, she was admitted to the hospital again for a re-evaluation at $9 \mathrm{mg}$ /day of oral GCS. Her mild $\operatorname{cog}$ nitive impairment remained unimproved on the MMSE (25/ 30) and WMS-R (verbal memory: 67, visual memory: 82 , general memory: 69, attention: 120, and delayed memory: < 50). Brain MRI showed no remarkable change in the interval from the previous examination (Fig. 1D), and FDG-PET revealed that the improvement in the hypermetabolism in the left posterior hippocampus had been maintained as well (Fig. 2C). We measured the SUV of FDG-PET and also analyzed the findings of previous FDG-PET examinations. The mean SUV was 4.38, and the maximum SUV was 5.04, indicating that the degree of hypermetabolism was almost the same as that just after the additional IVMP therapy. An overnight long-term vEEG revealed two subclinical seizures arising from the right mid-posterior temporal area and six from the left temporal area (Fig. 3).

Given the symptoms and examination results, we considered the degree of "smoldering" to be almost the same as it had been six months earlier. At present, the disease is well controlled with oral GCS at $9 \mathrm{mg} /$ day without further relapse.

\section{Discussion}

The patient developed HE manifesting as limbic encepha- 


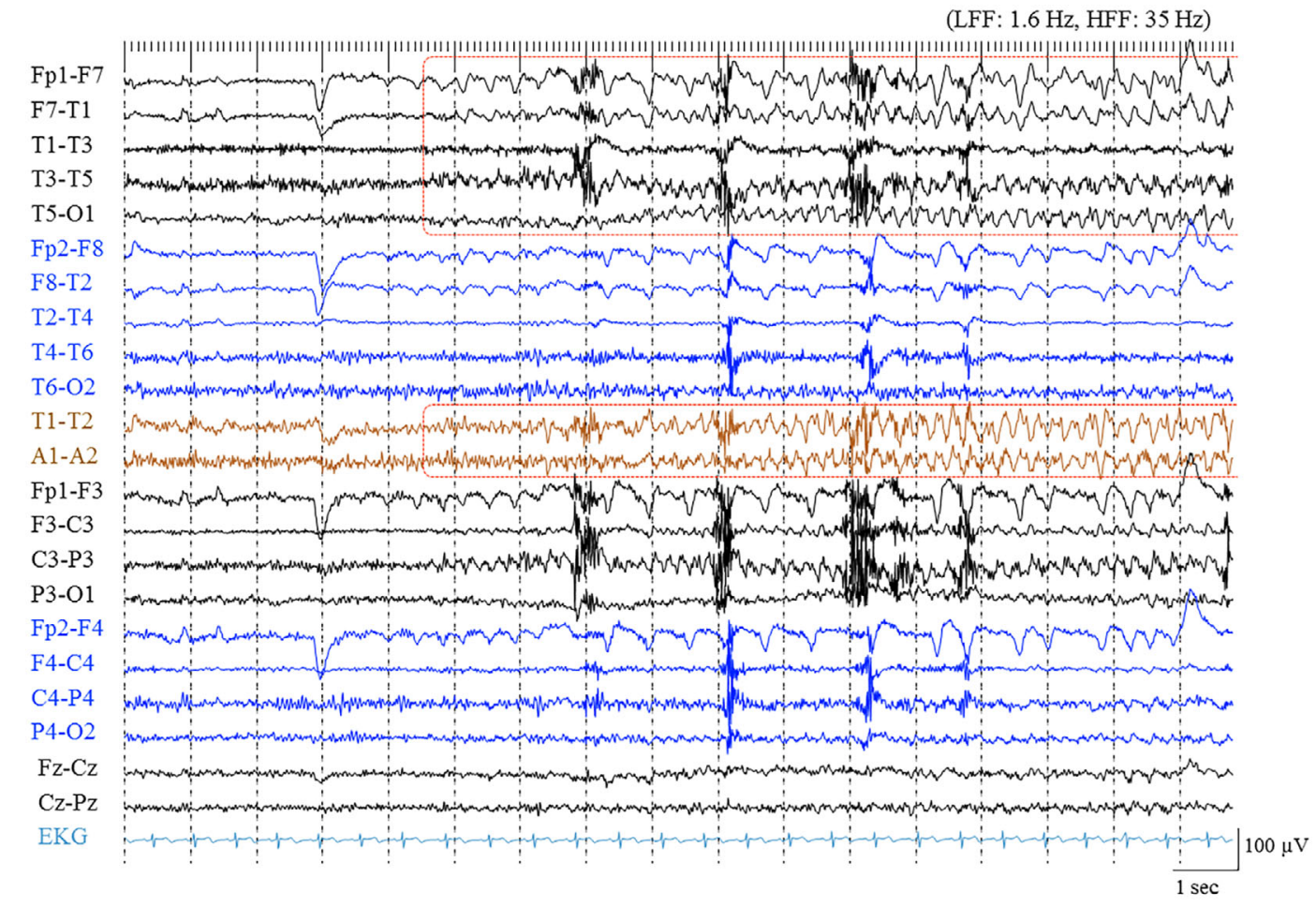

Figure 3. Subclinical seizures on an overnight video-electroencephalogram (vEEG). An overnight vEEG performed 1 year after the clinical onset showing subclinical seizure patterns both in the left temporal area (6 times in 24 hours) and right temporal area ( 2 times in 24 hours). A representative subclinical seizure pattern arising from the left temporal area is presented (red dotted lines). LFF: low-frequency filter, HFF: high-frequency filter

litis with persisting chronic symptoms and abnormalities in laboratory, neurological, imaging, and EEG examinations, suggesting a "smoldering" pathophysiology. This case raises two important new clinical considerations: 1) HE can present as "smoldering" limbic encephalitis, and 2) FDG-PET and EEG can reflect the disease activity on HE presenting as limbic encephalitis as previously reported in patients with other types of autoimmune limbic encephalitis $(9,10)$.

HE was first reported by Brain et al. in 1966, and about 300 cases have been reported subsequently $(1,2)$. For its diagnosis, anti-TPO antibody and the exclusion of other forms of encephalitis are essential $(16,17)$. Based on its clinical course, HE is classified into acute and diffuse types. The diffuse type often manifests as progressive dementia (18). In some previous cases of $\mathrm{HE}$, the late initiation of treatment was considered to increase the risk of residual memory impairment and/or hippocampal atrophy $(5,19)$. In 2002, Yoneda et al. found anti-NAE antibody to be a specific antibody of HE (7). Furthermore, in 2017, they reported a subtype of HE presenting as limbic encephalitis (8). Based on their findings, the case reported herein should be included in this subtype of HE because of the observations of bilateral high-intensity lesions in the mesial temporal lobes on brain MRI and the presence of symptoms associated with dysfunction of the limbic system, such as memory impairment and consciousness disturbance.

Anti-LGI1 antibody limbic encephalitis is often followed by a mild but long-lasting residual functional disorder, probably due to inflammation (20). Indeed, ongoing functional complications were observed in two cases of "smoldering" anti-LGI 1 antibody encephalitis reported by our group $(9,10)$. Similarly, our case of HE presented with left posterior hippocampal steroid-responsive hypermetabolism on FDG-PET and subclinical seizures on an EEG, both of which seemed to reflect persistent inflammation. Some types of autoimmune encephalitis emerge as severe refractory status epilepticus, such as new-onset refractory status epilepsy (NORSE), which shows refractory clinical seizures (21). Compared with the severe encephalitis that is refractory and usually causes a decline in activities of daily living, our concept of "smoldering encephalitis" at this point is different in that it causes mild or no clinical seizures and very gradual or no higher brain and/or cognitive dysfunction. Patients with "smoldering encephalitis" present with "smoldering" inflammation, as suggested by subclinical seizure patterns and epileptiform discharges on EEGs and hypermetabolism on FDG-PET. We therefore considered the clinical course of the present HE patient to be "smoldering" encephalitis with hippocampal atrophy, which is often seen in cases of anti-LGI1 antibody encephalitis.

Recently, the clinical importance of FDG-PET in autoimmune encephalitis, including anti-LGI1 antibody encephalitis, has been emphasized (22). Although whether hypermetabolism itself is due to inflammation or epileptic activity is 
controversial, FDG-PET has been considered to be more sensitive than MRI in terms of detecting inflammation (11-15). In HE patients, FDG-PET has been evaluated only in two case reports. One case showed diffuse unilateral temporal hypometabolism and the other medial and superior dorsolateral frontal hypometabolism, and both were responsive to steroids $(23,24)$. In contrast, our HE patient showed steroid-responsive focal hypermetabolism, suggesting active inflammation with an underlying mechanism of autoimmune abnormalities observable on FDG-PET, although we could not deny the possibility of hypermetabolism due to residual epileptic activity. In addition, the chronological decrease in the SUV values of this lesion may be partially explained by the partial volume effect, considering the small size of the focal lesion presenting with gradual atrophy. The differences between the present and previous cases might be due to the varied clinical characteristics of HE; our case showed limbic encephalitis, while most cases of HE present with cortical and/or subcortical dysfunction. In "smoldering" limbic encephalitis of HE, hypermetabolism on FDG-PET may reflect the disease activity.

Improving the frequent seizure patterns on EEG commonly seen in anti-LGI1 antibody encephalitis is thought to be important for resolving memory impairment $(9,25,26)$. Thus, treating subclinical seizures in autoimmune encephalitis may contribute to long-term memory improvement. HE generally presents with various EEG abnormalities, such as generalized slow waves, lateralized periodic discharges, triphasic waves, and temporal epileptiform activities $(18,27-30)$. Clinical symptoms and slow waves on EEG generally have been considered to correlate with the disease activity in conventional types of HE (31). In contrast, the correlation between the epileptiform discharges and disease severity in HE is still unclear. As in our case, despite improvements on FDG-PET, the re-evaluation of the long-term vEEG showed no obvious improvement with regard to the seizures with chronic lateralizing variation. Given that the EEG seizure patterns persisted while hypermetabolism on FDG-PET was relatively improved, it is uncertain whether the residual memory impairment in this particular HE patient was due to the sequelae of encephalitis or to "smoldering" of encephalitis. The long-term follow-up of this patient will help clarify this issue.

In previous cases of anti-LGI1 antibody encephalitis, early intensive treatment correlating with decrements in the titer of the antibody resulted in a good prognosis, but the optimal treatment regimen and period have not been defined (32). In both anti-LGI1 antibody encephalitis and HE, the diagnosis should be made early and appropriate treatment introduced in order to prevent persistent memory impairment as sequelae, as a delayed diagnosis and treatment are common risk factors for a poor functional outcome $(5,19,32)$. The hypermetabolism on FDG-PET in our HE case presenting with "smoldering" limbic encephalitis might have been correlated with the disease activity. Treatments targeting hypermetabolism on FDG-PET might lead to a good memory prognosis in not only anti-LGI1 antibody encephalitis but also "smoldering" limbic encephalitis of HE. Anti-LGI1 antibody encephalitis and HE presenting with limbic encephalitis share some characteristics in that they are both steroid-responsive with little association with tumors, although HE presenting with limbic encephalitis tends to show female predominance and more CSF abnormalities than anti-LGI1 antibody encephalitis $(8,33)$. LGI1 is the neural membrane protein in the VGKC complex, and its dysfunction has been estimated to lead to neural activity impairment (10). NAE is a protein identified by a proteome analysis using the serum of HE patients, and its pathophysiological role in HE remains unclear (7). Further investigations will be needed to clarify the patho-mechanistic similarities between these two types of limbic encephalitis.

Thus, the accumulation of further cases is needed in order to assess the capability of FDG-PET and long-term vEEG as surrogate markers of disease activity in "smoldering," autoimmune limbic encephalitis of HE.

\section{Author's disclosure of potential Conflicts of Interest (COI).}

Akio Ikeda: Honorariam, Eisai and UCB Japan; Research funding, GlaxoSmithKline, Nihon Kohden Corporation, Otsuka Pharmaceuticals and UCB Japan.

\section{Financial Support}

We would like to thank the Masakuni Kameyama Memorial Fund.

\section{References}

1. Brain L. Hashimoto's disease and encephalopathy. Lancet 288: 512-514, 1966.

2. Zhou JY, Xu B, Lopes J, Blamoun J, Li L. Hashimoto encephalopathy: literature review. Acta Neurol Scand 135: 285-290, 2017.

3. Ghika-Schmid F, Ghika J, Regli F, et al. Hashimoto's myoclonic encephalopathy: an underdiagnosed treatable condition? Mov Disord 11: 555-562, 1996.

4. Endres D, Perlov E, Stich O, Tebartz van Elst L. Steroid responsive encephalopathy associated with autoimmune thyroiditis (SREAT) presenting as major depression. BMC Psychiatry 16: 184, 2016.

5. Galluzzi S, Geroldi C, Zanetti O, Frisoni GB. Hashimoto's encephalopathy in the elderly: relationship to cognitive impairment. J Geriatr Psychiatry Neurol 15: 175-179, 2002.

6. Endres D, Perlov E, Riering AN, et al. Steroid-responsive chronic schizophreniform syndrome in the context of mildly increased antithyroid peroxidase antibodies. Front Psychiatry 8: 64, 2017.

7. Yoneda M, Fujii A, Ito A, Yokoyama H, Nakagawa H, Kuriyama M. High prevalence of serum autoantibodies against the amino terminal of $\alpha$-enolase in Hashimoto's encephalopathy. J Neuroimmunol 185: 195-200, 2007.

8. Kishitani T, Matsunaga A, Ikawa M, et al. Limbic encephalitis associated with anti-NH2-terminal of $\alpha$-enolase antibodies: a clinical subtype of Hashimoto encephalopathy. Medicine (Baltimore) 96: e 6181, 2017.

9. Kanazawa K, Matsumoto R, Shimotake A, et al. Persistent frequent subclinical seizures and memory impairment after clinical remission in smoldering limbic encephalitis. Epileptic Disord 16: 312-317, 2014. 
10. Nakaoku Y, Maki T, Kanazawa K, et al. A case of smoldering anti-leucine-rich glioma-inactivated 1 (LGI1) antibody-associated limbic encephalitis with faciobrachial dystonic seizure. Clin Neurol 53: 706-711, 2012 (In Japanese, Abstract in English).

11. Heine J, Prüss $H$, Bartsch $T$, et al. Imaging of autoimmune encephalitis - relevance for clinical practice and hippocampal function. Neuroscience 309: 68-83, 2015.

12. Baumgartner A, Rauer S, Mader I, Meyer PT. Cerebral FDG-PET and MRI findings in autoimmune limbic encephalitis: correlation with autoantibody types. J Neurol 260: 2744-2753, 2013.

13. Fakhoury T, Abou-Khalil B, Kessler RM. Limbic encephalitis and hyperactive foci on PET scan. Seizure 8: 427-430, 1999.

14. Barboriak DP, Edward R. Limbic imaging. Psychiatry interpers Biol Process 170: 1659-1660, 1998.

15. Kassubek J, Juengling FD, Nitzsche EU, Lücking CH. Limbic encephalitis investigated by 18FDG-PET and 3D MRI. J Neuroimage 11: 55-59, 2001.

16. Graus F, Titulaer MJ, Balu R, et al. A clinical approach to diagnosis of autoimmune encephalitis. Lancet Neurol 15: 391-404, 2016.

17. Mijajlovic M, Mirkovic M, Dackovic J, Zidverc-Trajkovic J, Sternic N. Clinical manifestations, diagnostic criteria and therapy of Hashimoto's encephalopathy: report of two cases. J Neurol Sci 288: 194-196, 2010.

18. Kothbauer-Margreiter I, Sturzenegger M, Komor J, Baumgartner R, Hess CW. Encephalopathy associated with Hashimoto thyroiditis: diagnosis and treatment. J Neurol 243: 585-593, 1996.

19. Segers K, Braconnier P, Corazza F, et al. Subacute cognitive deterioration with high serum anti-thyroid peroxidase antibodies: two cases and a plea for pragmatism. Psychogeriatrics 13: 175-179, 2013.

20. Malter MP, Frisch C, Schoene-Bake JC, et al. Outcome of limbic encephalitis with VGKC-complex antibodies: relation to antigenic specificity. J Neurol 261: 1695-1705, 2014.

21. Nicolas G, Brandon FP, Vincent A, et al. New-onset refractory status epilepticus. Neurology 85: 1604-1613, 2014.

22. Morbelli S, Arbizu J, Booij J, et al. The need of standardization and of large clinical studies in an emerging indication of [18F] FDG PET: the autoimmune encephalitis. Eur J Nucl Med Mol Imaging 44: 353-357, 2017.

23. Pari E, Rinaldi F, Premi E, et al. A follow-up 18F-FDG brain PET study in a case of Hashimoto's encephalopathy causing drugresistant status epilepticus treated with plasmapheresis. J Neurol
261: 663-667, 2014.

24. Endres D, Vry MS, Dykierek P, et al. Plasmapheresis responsive rapid onset dementia with predominantly frontal dysfunction in the context of Hashimoto's encephalopathy. Front Psychiatry 8: 1-7, 2017.

25. Navarro V, Kas A, Apartis E, et al. Motor cortex and hippocampus are the two main cortical targets in LGI1-antibody encephalitis. Brain 139: 1079-1093, 2016.

26. Aurangzeb S, Symmonds M, Knight RK, Kennett R, Wehner T, Irani SR. LGI1-antibody encephalitis is characterised by frequent, multifocal clinical and subclinical seizures. Seizure 50: 14-17, 2017.

27. Santoro D, Colombo I, Ghione I, et al. Steroid-responsive Hashimoto encephalopathy mimicking Creutzfeldt-Jakob disease. Neurol Sci 32: 719-722, 2011.

28. Rodriguez AJ, Jicha GA, Steeves TDL, Benarroch EE, Westmoreland BF. EEG changes in a patient with steroidresponsive encephalopathy associated with antibodies to thyroperoxidase (SREAT, Hashimoto's encephalopathy). J Clin Neurophysiol 23: 371-373, 2006.

29. Castillo PR, Boeve BF, Westmoreland BF, Scha B. EEG findings in steroid-responsive encephalopathy associated with autoimmune thyroiditis. Clin Neurophysiol 114: 32-37, 2003.

30. Henchey R, Cibula J, Helveston W, Malone J, Gilmore RL. Electroencephalographic findings in Hashimoto's encephalopathy. Neurology 45: 977-981, 1996.

31. Marshall GA, Doyle JJ. Long-term treatment of Hashimoto's encephalopathy. J Neuropsychiatry Clin Neurosci 18: 14-20, 2006.

32. Wong SH, Saunders MD, Larner AJ, Das K, Hart IK. An effective immunotherapy regimen for VGKC antibody-positive limbic encephalitis. J Neurol Neurosurg Psychiatry 81: 1167-1169, 2010.

33. Lai M, Huijbers MGM, Lancaster E, et al. Investigation of LGI1 as the antigen in limbic encephalitis previously attributed to potassium channels: a case series. Lancet Neurol 9: 776-785, 2010.

The Internal Medicine is an Open Access journal distributed under the Creative Commons Attribution-NonCommercial-NoDerivatives 4.0 International License. To view the details of this license, please visit (https://creativecommons.org/licenses/ by-nc-nd/4.0/).

(C) 2019 The Japanese Society of Internal Medicine Intern Med 58: 1167-1172, 2019 